\title{
A generic Integrated Spatial Decision Support System for urban and regional planning
}

\author{
$\underline{\text { H. van Delden }}{ }^{\text {a }}$ and J. Hurkens ${ }^{\text {a }}$ \\ ${ }^{a}$ Research Institute for Knowledge Systems, Witmakersstraat 10, 6211 JB Maastricht, The Netherlands \\ Email: hvdelden@,riks.nl
}

\begin{abstract}
The proposition that land use dynamics are complex and exhibit self-organising behaviour may seem at odds with the prima facie assumption that planners and policy-makers have a decisive influence on future land use. In reality, this is not a contradiction, but rather a challenge for model developers to recognize disparate types of drivers and to look at autonomous behaviour and (spatial) planning as an integral part of land use dynamics. Furthermore, experience learns that in order to support decision making and/or policy analysis we have to bridge the science-policy gap by ensuring that i) models incorporate policy-relevant drivers and provide policy-relevant output in a comprehensible form, and ii) buy-in from users is achieved by involving them in the development process.
\end{abstract}

This paper presents a generic integrated spatial decision support system (ISDSS) for simulating urban and regional dynamics. The system aims to support long-term integrated policy development and planning by taking into account social, cultural, environmental and economic developments in an integrated fashion. It provides an easy-to-use interface targeting both policy analysts and scientists. The system is built using a software environment for dynamic spatial modelling and includes a library with models from various disciplines: economics, demographics, regional interaction, land use and transport. It is generic enough to be configured and applied to new regions without the need for additional software development: models and indicators can freely be selected and parameterised to achieve maximum relevance for addressing the issues in the region under study.

When the generic ISDSS does not fulfil all user requirements, the system can be extended with other models, which may be existing, region-specific models or models developed to simulate particular relevant processes. In such cases, software development and adaptations of the user interface are required for the incorporation of these models, resulting in a tailor-made ISDSS. Such a system includes models from a wider range of disciplines and can as such (additionally) incorporate components for virtually any process that can be modelled. Current applications include integrations of climate, hydrology, water quality, vegetation dynamics, biodiversity and ecosystem services models. All these models are dynamic simulation models with temporal resolutions varying between minutes and years and a time horizon of 20 to 50 years into the future. While some socio-economic models incorporated are non-spatial, most others are spatially explicit. Most tailor-made systems include different spatial hierarchies and resolutions within one integrated model.

In this contribution we will focus on the development history of the generic ISDSS and demonstrate its applicability to a diverse range of regions worldwide. Furthermore we will show how parallel development trajectories of tailor-made systems and a generic tool can co-exist and how developments in one system can be reused in other systems. We will demonstrate how the development of all these systems has been made possible by building upon experience and reusing models, software and design, leading to the evolution of a (more) generically applicable framework. We will discuss the prerequisites to achieving this high level of reusability on various levels: model integration, ISDSS development and user interface design.

From the above applications we have learned the following lessons: (1) ISDSS development is a joint effort of users, scientists and IT-specialists, (2) In ISDSS development, software development is facilitating, not leading, (3) Conceptual challenges regarding model integration cannot be solved by technical solutions alone, (4) The need for software development in various phases of the development and use of an ISDSS can and should be minimized, (5) Reusable concepts and components allow for efficient development of new ISDSS, although genericity remains a trade-off, (6) Funding mechanisms should be accounted for in planning longterm ISDSS development and implementation trajectories.

Keywords: Integrated Spatial Decision Support System; Model integration; Policy support; Land use dynamics; Modelling framework 


\section{INTRODUCTION}

A closer look at the drivers of land use change makes it apparent that land use dynamics are driven by a combination of processes operating at various spatial and temporal scales (Veldkamp and Lambin, 2001; Verburg et al., 2004). Looking at urban environments for example, there is interaction between cities, between neighbourhoods within a city and between individual actors residing in those neighbourhoods, although none of these processes operate in isolation. Bottom-up as well as top-down interactions play a crucial role in the overall dynamics. Socio-economic developments at macro level impact on the demand for residential, industrial and commercial locations, while the availability of suitable locations and the actual spatial configuration in turn impact on the overall socio-economic developments. When looking at rural regions we see important interactions between socio-economic and biophysical processes (Oxley et al., 2004 and Van Delden et al., 2007). Farmers use natural resources for their agricultural practice and in turn have an impact on the natural environment and its resources. All of these interacting processes may or may not operate on the same spatial and temporal scale. For example, farmers make a decision on what crop to grow on a yearly basis, while the process of erosion often takes place during a few minutes.

The most important drivers for land use change appear to be similar over different geographic locations and different periods in time (Van Delden et al., 2009a). However, this usually only holds true on an abstract level and becomes less accurate when focusing on the details. It is widely acknowledged that existing urban activities are an important attracting factor for the allocation of new urban activities (White and Engelen, 1993). Still, a closer look at this topic shows that there are vast differences between the clustering of urban land uses in different regions. This leads us to conclude that it is feasible to have a general framework that incorporates the main drivers for land use dynamics, due to the similarity of main drivers and processes, but that every application within such a framework needs to be calibrated to the specific dynamics of the location and period in time.

Over the past years various integrated models and ISDSS have been developed aiming to support policy analysis in the field of land use and spatial planning (Van Ittersum et al., 2008; Sieber et al., 2010). Besides the incorporation of the relevant processes these systems need to connect to the contents and process of the policy practice. Until now, most systems are tailor-made products that require software developers to configure the integrated model, or are loosely-coupled systems which require modellers to run the individual components. Each of these options is very time consuming in system development and use. The latter also offers little flexibility to the policy analyst.

This paper presents Metronamica, a generic ISDSS for urban and regional planning, which has been applied to a range of cities, rural environments and coastal zones worldwide. The system can be applied to a region of choice without new software development and offers users the flexibility to select one or more models from the model library and apply them at the resolution of choice. Metronamica includes a dual user interface for policy analysts and modellers and thus provides a specialised access for the two main types of users.

Section 2 gives an overview of the Metronamica ISDSS and presents its main components. Section 3 describes its development trajectory while pointing out how various components of the system have evolved over time and how tailor-made systems contribute to the development of the generic ISDSS. Section 4 gives an overview of the main elements of reusability in the development of ISDSS. Finally, section 5 concludes with some lessons learned in the development, use and maintenance of ISDSS in practice and discusses some challenges lying ahead.

\section{METRONAMICA, A GENERIC INTEGRATED SPATIAL DECISION SUPPORT SYSTEM}

Metronamica (RIKS, 2011; www.metronamica.nl) is a generic forecasting tool for planners and policy analysts to simulate and assess the integrated effects of policy measures on urban and regional development. The system interactively simulates the impact of a variety of external influences (e.g. macro-economic changes, population growth, etc.) and policy measures (e.g. land use zoning, conservation policies, densification policies, etc.) on the regional development of a city, region, country or continent. With the integrated scenario support what-if analyses can be performed that help evaluate alternative plans under various external conditions.

Metronamica is developed using the Geonamica software environment (Hurkens et al., submitted) and includes a model library containing a range of models from various disciplines: land use, regional interaction, transport, economics and demographics. Applications can be set up with one, two or three spatial levels depending on their scope. Spatial resolution at local level varies for current applications between $25 \mathrm{~m}$ and $1000 \mathrm{~m}$. Temporal resolution is a year. Temporal horizon is 20 to 50 years into the future. 


\subsection{Model Components}

The models that are incorporated in Metronamica simulate activities that take place at three spatial scales: global, regional and local, where global refers to the entire simulated area. At global level, a macro-economic model is tied with an age-cohort model that simulates structural demographic changes and population levels. The age-cohort model incorporates immigration patterns and provides the labour force supply; the latter are used as an input for the economic model. Economic conditions, in return, have an impact on migration and mortality rates. Figures for population and jobs in main economic sectors are used as an input for the regional model.

At the regional level, socio-economic changes take place based on the relative attractiveness of regions and the costs required to travel from one region to another. These costs are provided by the transport model that uses information from the regional and local models to generate trips and calculate the speed, intensity and congestion on the network. This provides the basis for the distribution of national growth as well as migration of jobs and people over regions and is furthermore input for the allocation of activities within the regions.

On the local level, land use demands from the regional model are allocated to grid cells based on several elements including local accessibility, physical suitability, zoning regulations and the attraction, repulsion and competition between different land use functions. Finally, the local bio-physical and socio-economic characteristics feed back into the attractiveness at the regional level and the land use configuration is used as an input for the production of trips in the transport model.

For each application the user is free to select one or a number of model components. Based on the selection of components, inputs for them come from other components or are defined as exogenous drivers. Details about the various configurations are described in section 2.2.

The different components and their interactions are schematised in the system diagram of Metronamica in Figure 1. The processes modelled in the components are described in the paragraphs below.

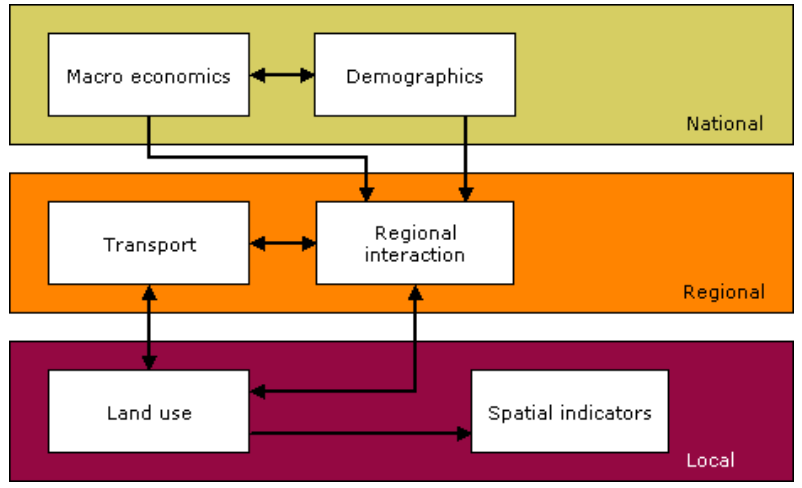

Figure 1. Metronamica system diagram showing the various components and their interactions.

\section{Demography}

Demographics are represented by an age-cohort model that generates annual population figures by gender and age, on the basis of births, deaths and net migration. Fertility, mortality, and net migration rates are each assumed to be characterized by trends. In addition, it can include features that capture the effects of changing economic conditions (as calculated by the macro-economic model). While the structural component represents the long-term underlying demographic trends, the economic component captures changes that may weigh in on demography due to changes in the wealth of the population. The economic component is particularly important in the case of migration, because the flow of migrants generally responds to changes in relative economic conditions. The demographic model provides the regional model with overall population figures on a yearly basis.

\section{Economy}

Macro-economic developments are modelled using a declarative econometric framework that allows users to include their own equations, variables, constants and data. As described above it provides input to the demographic model (the wealth of the country or region). Moreover, it uses the labour force as calculated by the demographic model. The macro-economic model provides the regional model with yearly figures on the total number of jobs in main economic sectors.

The choice for a declarative modelling framework means that region-specific econometric models can be used or developed, and easily incorporated in the system. E.g. in applications for Italy, Spain and Portugal models have been used that were set up in Excel. The equations were extracted using a macro in Excel and the data copied to prevent human error in the transition. This approach also makes it easier to adapt the model structure (i.e. the incorporated equations) when new scenarios or new indicators need to be developed or when new economic data becomes available, which experience has shown to happen at a faster rate than for other disciplines. 


\section{Regional interaction}

The regional interaction model simulates the migration of activity between regions and distributes the demographic and economic growth from the respective models over the regions. It uses the concept of gravity or spatial interaction modelling (Fotheringham and O'Kelly, 1989), in which interregional migration flows and overall growth/decline of activity (i.e. people and jobs) are modelled based on each region's attractiveness and the travel costs (or distances) between regions. The attractiveness of each region is determined based on the existing levels of activity in each region and factors from the local level, such as accessibility and land use conditions.

The measure of distance that we use in the spatial interaction model is very important. In fact, it is the determining factor of this model for the accuracy with which migration flows can be modelled. Without the transport model, Euclidian distances are used in this calculation. When the transport model is included, generalised travel costs are used in the calculation instead, which provides us with a better measure of distance (Fotheringham et al., 2000).

We have stated above that the levels of activity form a restriction on the land use demands of the local model. To be more precise, the level of activity is converted to a number of cells that needs to be allocated in a region. Cell-productivity expresses the average amount of activity in a sector per cell.

\section{Transport}

The transport model incorporated in Metronamica is based on a classical four step approach: productionattraction, distribution, modal split and assignment (Ortuzar and Willumsen, 1994). Like the regional interaction model it works at regional level, although its regions are transportation zones. They are generally smaller than the regions mentioned above and their sizes depend on the activity that can be found within the zone. Since urban and rural areas have different characteristics and behaviour regarding transport, a classification of the urbanization level of the different transport zones is used throughout the model.

In its first step, the model calculates the production of trip origins and destinations from each zone to each other zone based on the activity levels in each zone, calculated as aggregates from the land use maps, and the travel behaviour of different groups in society. In the distribution and modal split steps, production and attraction levels in different zones will be linked together to form trips. Actors select their destinations and their mode of transport as a function of the associated generalised costs. It takes time, however, for actors to change their behaviour and preferences. Therefore a major factor determining the selection of destinations and modes are the existing transport patterns. It is here where we have made a modification to the classical equilibrium based transport model in favour of a more dynamic approach. The result of the distribution and modal split steps is an origin-destination matrix (OD-matrix) specifying trips for each transport motive. In the assignment step, the car trips between each pair of zones are routed over the transport network using the shortest path, which is measured using generalised costs. The result of the assignment step is the allocation of all trips over the road network and the calculation of the intensity, congestion and travel speed on each road element. Average generalised costs between each pair of zones will be used in the distribution and modal split steps in the next time step.

\section{Land use}

The land use model operates at local level and uses a grid of cells, varying between $25 \mathrm{~m}$ and $1000 \mathrm{~m}$ in size depending on the scope of the model. A cellular automaton (CA) based land use model is used to determine the state of a cell within the overall growth for each of the regions calculated by the regional model (White and Engelen, 1993). Changes in land use at the local level are driven by four important factors that determine the potential for each location for each actor (see also Figure 2):

- $\quad$ Physical suitability, represented by one map per land use function modelled. The term suitability is used here to describe the aptness of a cell to support a particular land use function and its associated activity.

- Zoning or spatial planning, represented by one map per land use function modelled. For different planning periods the map specifies which cells can and cannot be taken in by the particular land use and how strict or flexible the various plans are.

- Accessibility, represented by one map per land use function modelled. Accessibility is an expression of the ease with which an activity can fulfil its needs for transportation, mobility and other facilities in a particular cell, based on the proximity to infrastructure networks. 
- Interaction rules, simulating the preferences of various actors for certain locations based on the land uses in the area surrounding the location, including their power to actually occupy the most desirable locations. For each land use function, a set of spatial interaction rules determines the degree to which it is attracted to, or repelled by, the other functions present in its surroundings.

If the potential is high enough, the function will occupy the location, if not, it will look for more attractive places. New activities and land uses invading a neighbourhood over time will thus change its attractiveness for activities already present and others searching for space. This process constitutes

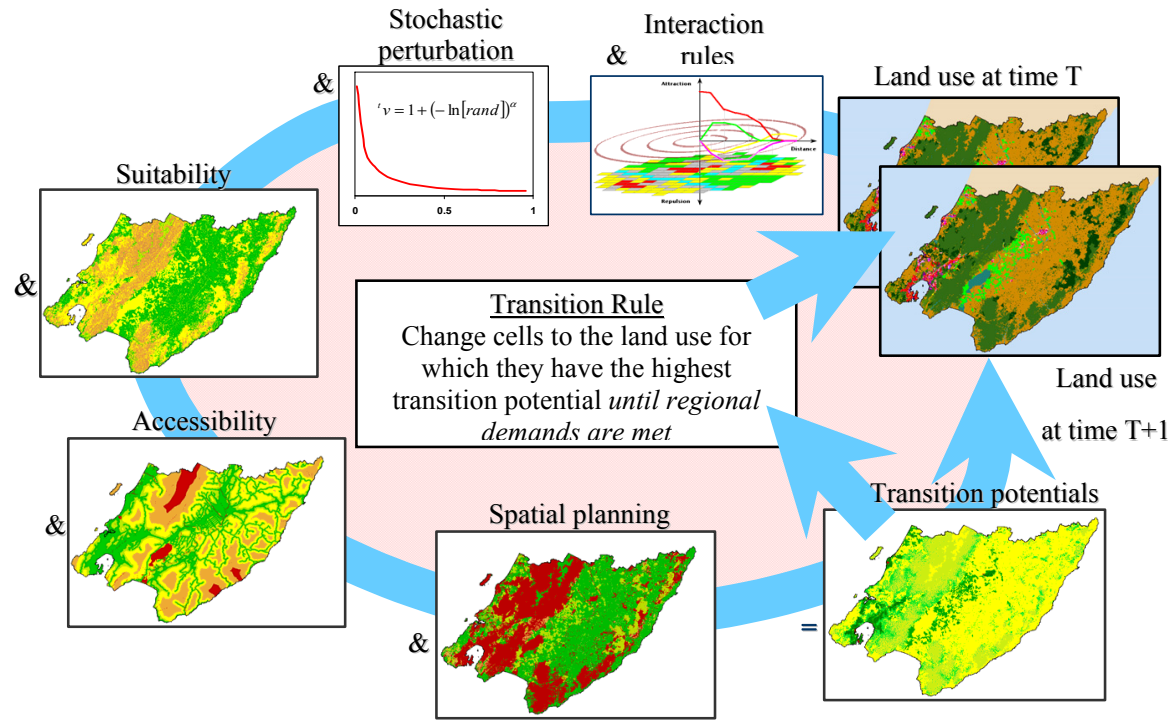

Figure 2: Main drivers of the Metronamica land use model as shown for an application to Greater Wellington, New Zealand. the highly non-linear character of this model.

\section{Indicators}

Metronamica includes a range of socio-economic and environmental indicators which can be selected and configured based on a selection of algorithms. A set of generically applicable indicators is predefined using a categorisation of the land use classes (e.g. urban, natural, etc.) to set reasonable parameter values. These parameters can be fine-tuned to provide better results. Examples of such indicators are the expansion of urban areas, habitat fragmentation and the distance from residential locations to the nearest recreation site. Other indicators can be added on demand by selecting one from a set of available algorithms, providing additional input data and adjusting model parameters. Examples of such indicators are urban development in areas prone to flooding - requiring a map indicating areas prone to flooding - and job potential, which is the ratio between the number of jobs and inhabitants - taken from the regional interaction model - in the vicinity of a residential location.

\subsection{User Interface}

An important task for the developer of an ISDSS is to bridge the gap from scientific tools to user-friendly systems, by creating a graphical user interface (GUI) that is easy to use and guides users in the steps that need to be taken to carry out a scenario or policy impact assessment study. In addition, as ISDSS often encompass complex models, the user interface should provide insight into the structure and functioning of the model and provide access to all relevant model inputs and outputs for updating the data, calibration and validation. Trying to incorporate both in one interface often leads to a malfunctioning system that is far from optimal for any user.

In the design of the Metronamica user interface we decided that the interface should be able to provide access to two different types of users: the policy analysts who use the system as part of their policy process and who carry out scenario and impact assessment studies with the model, and the scientists or modellers who can update the underlying data and parameters and possibly even the model equations. For the latter group of users we created the modeller interface where elements are grouped per model; each individual model has its own access point through the system diagram (see Figure 1). Access to settings for the policy user is structured according to their logical function in the policy interface. On a high level, access is organized by 
the steps that a user takes to carry out an impact assessment analysis: configure drivers, create integrated scenarios, run the simulation, review output through the indicators and do comparative analysis. Zooming in on those parts, we grouped settings and outputs by their type and their domain; for example all economic policy measures together, all external factors together, all ecological indicators together etc. An example of the policy interface is provided in Figure 3.

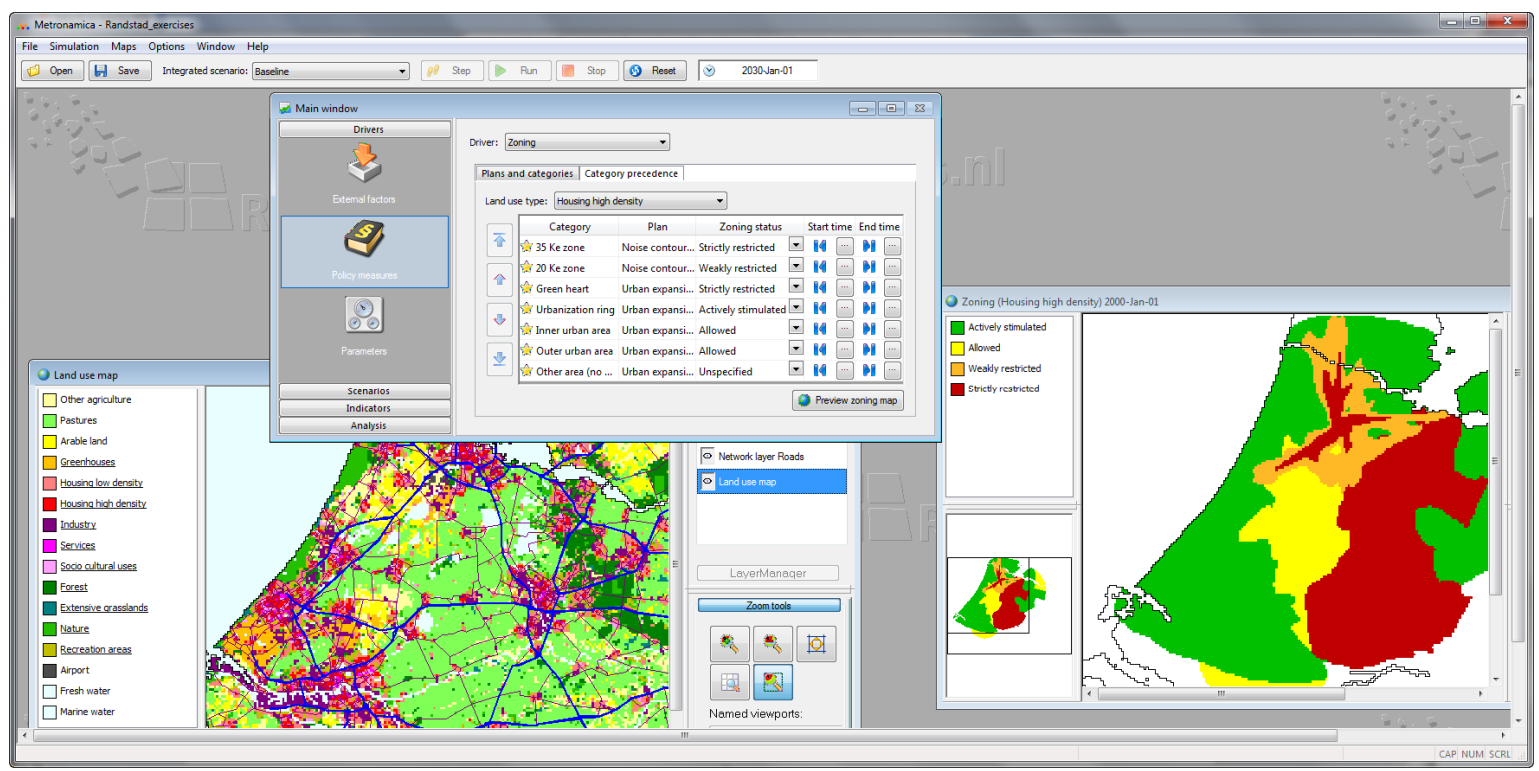

Figure 3: User interface of Metronamica showing the policy interface in the main window, on the left hand structured according to steps for policy impact assessment.

\subsection{Applying the System to a New Region}

Metronamica includes a wizard that supports users in setting up an application for a new region. The wizard follows a series of steps in which the desired model configuration is selected and input data is imported and interpreted by the user. The wizard checks the data for any inconsistencies to prevent errors in the use of the system later on. When all steps are completed, the wizard configures the application with sensible initial parameter values, which can then be calibrated to mimic the characteristics of the study area. Only strictly required input data to set up a minimally functional application needs to be provided in the wizard. This makes it easy to set up new application, while the normal user interface can be used to incorporate additional data or adjust parameters thus providing transparency in the calibration parameters and data incorporated.

Important choices that a user makes while using the wizard are: the selection of models, the area extent, the locations of the regions and transport zones, the resolution at local level, the national and regional activities, the local land uses and the transport motives. Basic data required to set up Metronamica include a region map, a land use map and a map for transport zones (in case the transport model is selected). All other data and parameters are included through the modeller interface after the application has been set up.

\section{DEVELOPMENT TRAJECTORY AND APPLICATIONS}

In the early nineties, RIKS developed a CA-based land use change model (White and Engelen, 1993), which was turned into a user-friendly ISDSS in the late nineties. As land use change has various drivers, over time requests came to include more drivers in a dynamic way and to create feedback loops between reciprocal processes. For each specific application, models were coupled to the land use model and incorporated in the software. Although this provided advancements in the scientific and technical integration of models as well as in the ISDSS development process as a whole, it created problems in the maintenance of those systems, as changes became harder to incorporate, due to an increasing amount of dependencies within the software. Both the software and user interface grew more complex as configuration options increased and a dichotomy arose between old and new features or components. Problems aggravated for those systems where land use was not included, such as WadBOS for Dutch coastal waters (Engelen et al., 2003) and the Lobster IBM (an 
individual based model for Canadian Lobster Fisheries, http://www.riks.nl/projects/Lobster-IBM) as solutions had to be found to 'hide' the land use components from the user interface.

The above problems resulted in the separation of the software environment, named Geonamica, and a range of model components. In 2005 the decision was taken to redesign the entire system. The software engine and the user interface elements became part of the Geonamica software environment, while the land use model and other models were placed in a library of models, some developed by RIKS, but most developed together with third parties. Well-tested, generically applicable components were placed in Metronamica, which is developed using Geonamica and a subset of models from the overall model library, as described in the previous section. The remainder of this section presents the past and ongoing development process of Metronamica.

\subsection{Design Choices and Continued Development}

In the development of Metronamica we have moved to a highly modular implementation of models in order to facilitate ongoing developments and support parallel versions of the model. Experience has shown that model integration often requires subtle changes to a model. In some cases, these changes cannot be made in a way that previous applications of the model remain valid. In such cases, a modular implementation helps, because a new component can be developed to replace an existing one, while previous applications can still use the old component. For this to work in practice, the granularity of model components should match the level at which changes affect the model, i.e. a change should require us to adapt a large part of a small number of components. If a change only requires us to adapt a small part of a component, this implies we need to duplicate relatively large amounts of code to achieve backwards compatibility. Conversely, if a change affects a large number of components, we have gained nothing from the overhead of this high level of modularity and would have been better off with larger components.

Developments of Metronamica are both demand and supply driven. On the one hand, they take place based on project specific needs or requests from people who use Metronamica by themselves. On the other hand, they are driven by the development team based on user feedback and scientific or technical advancements coming from their expertise in working in this domain and more specifically in working with the software. Funding for ongoing development comes from license fees, research projects and the development of tailormade systems for clients. Concrete user enhancements are often funded through the latter funding stream, while ongoing development on the software environment, scientific improvement of the various model components and the development of new model components mainly takes place through research funding and license fees. Based on user needs and developer ideas a strategy is maintained for the future development of Metronamica and based on this strategy concrete funding options are sought.

\subsection{Making Use of Developments in Tailor-made Systems}

An important part of the initial and ongoing development of Metronamica has been and still is taking place through the development of tailor-made systems that are developed using Geonamica, the Metronamica model library and additional models. Examples of tailor-made systems are provided below together with their aim and the included models. The subsections below the overview detail some of the specific developments that have taken place through these tailor-made systems and which were subsequently incorporated in Metronamica once they reached a sufficient level of maturity.

- RamCo: focusing on coastal zone management in South-west Sulawesi, Indonesia. Includes components for land use, coastal waters and river basin management (De Kok and Wind, 2002).

- Xplorah aiming to support integrated planning on the island of Puerto Rico (Van Vliet et al., 2009; Van Delden et al., 2010a). Includes components for land use, regional interaction, transport, economics, demographics and climate change.

- The MedAction PSS and its successor the DeSurvey IAM, aiming to provide support to regional development and desertification and having a main focus on sustainable farming, water resources and land degradation in arid and semi-arid regions (Van Delden et al., 2007; Van Delden et al., 2009b). Include components for land use, climate change, hydrology, erosion, sedimentation, salinisation, vegetation growth, farmers' decisions, water resource management and economics.

- MOLAND focusing on urban planning, with applications to a range of cities and city regions, mainly in Europe (Lavalle et al., 2003; Barredo et al., 2003). Includes components for land use, regional interaction and transport. 
- The LUMOCAP PSS aiming for an integrated assessment of (agricultural) policies on the European land use and landscape (Van Delden et al., 2010b). Includes components for land use, agricultural economics, climate change and dynamic suitability.

- WISE, A-ISE and GW-ISE (Waikato, Auckland and Greater Wellington Integrated Scenario Explorers) aiming to support the development of a strategic vision for New Zealand regions by taking into account social, environmental and economic well-being (Rutledge et al., 2008; Huser et al., 2009; and Van Delden et al., 2011a). The systems include some or all of the following components: land use, demographics, ecological economics, hydrology, water quality, climate and biodiversity.

\section{From specific to generic model components}

The transport and economic models both are examples of models that were originally developed for specific users and over time became generic components. Originally, these models where incorporated in tailor-made ISDSS based on specific user requests for integration with the land use model. In subsequent projects these models were enhanced and included into other tailor-made systems, while the improvement were also shared with the original users who could in this way benefit from the ongoing developments. As further requests for these models came, the decision was made to develop them into generic components that could be applied to new regions without further programming and at this stage they were incorporated into Metronamica.

\section{Enhancement of existing model components}

Early in the development of the land use model, spatial planning was included as a driver for land use change through the incorporation of a zoning map indicating where land use developments were allowed. Over time, it became clear that both the user-friendliness and the scope of this driver were too limited for effective use. Based on findings from a range of users, we decided to develop a zoning tool that would allow for an interpretation and integration of spatial plans (from a GIS) directly in the land use model. The scope of this driver was also expanded by creating a range of possibilities for interpreting them based on the strictness or flexibility of the original plan. The new zoning tool thus offers additional flexibility in the interpretation of the plans and an increased transparency through the incorporation of the underlying data and the explicit interpretation thereof. After its first introduction, further enhancements of the zoning tool have been made as a result of its application in tailor-made systems. When this tool reach maturity it was incorporated as a generic component in Metronamica.

\section{Further development of the user interface}

The larger the number of models that were incorporated in the tailor-made systems, the harder it became to keep these systems user-friendly. Initially, when the GUI was organised through a system diagram, policy analysts did not have a proper overview of the total set of drivers and indicators and experienced difficulties in managing scenario runs as there were so many different inputs and parameters they could change. Modellers on the contrary were very satisfied with the interface that was structured according to model components and that allowed a large flexibility in updating data and parameters. Because we wanted to keep the interface that was working for the modellers, while improving the interface for the policy analysts, we developed a dual user interface as described in section 2.2. As part of the policy interface we included a scenario manager that allows for a hierarchical construction of scenarios by creating and storing a range of sub-scenarios (a range of input values) for each driver and subsequently integrating these sub-scenarios into integrated scenarios using the scenario manager.

\section{Development of the software environment}

The development of tailor-made systems raised the need for a model-agnostic software environment for model development, model integration and ISDSS development. By capturing application logic and software components that are not central to the model itself, the Geonamica software environment helps to maximise reuse of implementation and design in developing and maintaining a wide variety of ISDSS in parallel (Hurkens et al., submitted). The shift from a model-specific application (which Metronamica originally was) to a model-agnostic framework upon which specific applications are built, has not been without effort. Many design choices in the model-specific software implementation have had to be revised in order to fit the generic framework based on fundamental modelling paradigms and common software design principles, such as a strict separation of code for the framework, user interface and models. However, this has paid off by offering a great increase in the reusability of developments, many of which can be implemented directly in the framework or using the framework, so that they can be reused in all projects with little to no need for additional software development for each specific application. Examples of such functionality are: user 
interface components, support for managing scenarios, support for sensitivity analysis, tools for management, dissemination and joint development of scenarios, and tools for comparison and auditing of model parameters.

Nowadays, Geonamica is a stand-alone software environment that contains no models itself, but can be customised to produce tailor-made or reusable ISDSS, such as those mentioned in this paper. It provides data structures and common functionality to implement models, a modelling and model integration framework based on the DEVS formalism (Zeigler et al., 2000) and a GUI environment and components to develop customised, user-friendly applications. Existing model implementations can be incorporated by creating a wrapper that exposes the interface of a Geonamica model component. Any model can be incorporated into Geonamica this way, whether it is available as an executable, a library (DLL), a web service, an implementation with an OpenMI compliant interface or as source code. The models that are incorporated in Metronamica are available as libraries that can be plugged into a Geonamica application without further software development. User interfaces are typically custom designed for each application based on a reusable structure and reusable components - see section 4.4.

\section{REUSABILITY}

Reusability offers great benefits in the efficiency with which ISDSS can be developed, applied and used. Moreover, building on existing work allows to further develop and perfection the components and the development process during each iteration round. Below we describe how we reuse various elements.

\subsection{Design and Development Methodology}

Based on our experience in ISDSS development we have developed a methodology that is followed in each project and development trajectory. A main lesson learnt from previous work is that DSS development is an iterative process that includes users, scientists and IT-specialists. The methodology provides guidance on how to involve the various groups, the important steps to take and the pitfalls and opportunities during the design and development process (Van Delden et al., 2011b). We have learned that following such a methodology improves both the quality of the ISDSS and the efficiency of the process.

\subsection{Software Environment for Model Integration}

For a model integration framework to be reusable it has to build on a strong theoretical foundation that specifies how model components can communicate in a way that guarantees a (technically) sound result. In many model integration frameworks, model components can expose inputs and outputs, which can be linked by the framework or the application programmer in order for these components to communicate with each other. However, when a model is to be used in an ISDSS, another kind of communication is required, namely to relay the user input to model components. As they require different behaviour, user interface components cannot be implemented as model components and, therefore, the model integration framework must offer a dedicated interface for user interface - model communication that guarantees model consistency.

For a model integration framework to be reusable it also has to be practical. This means that the work required to implement models (or adaptors to existing model implementations) should be very limited. The framework should implement common functionality where possible and the interface that must be implemented by each model component must be minimal. It also means that the overhead (particularly in terms of running speed) imposed by the framework should be minimal. Finally, a model integration framework should not unnecessarily limit the scope of the models that can be included, i.e. it should support a large number of modelling paradigms. The contradicting nature of these requirements implies that design choices have to be made in favour of one or the other. E.g. the Geonamica software environment is designed to maximise performance and minimise the amount of programming required to develop discrete time simulation models. Consequentially, it is hard to incorporate other modelling paradigms in a Geonamica application, let alone integrate these kinds of models.

Another balance that must be made is in the flexibility - in terms of model integration - that is offered to users of an application developed with the model integration framework. This is a gliding scale, where on one end we find so-called component based frameworks (Muetzelfeldt, 2004) that treat model components as black boxes and where the incorporated models and model links are typically hard coded, thus leaving no flexibility to users to change the configuration of an integrated model unless this has been foreseen in the software implementation. On the other end, we find declarative modelling frameworks (Muetzelfeldt and Massheder, 2003), in which the entire model is specified in run-time e.g. as a set of equations or using system 
diagrams that can be created by the user. These frameworks offer a lot of flexibility, but pay the price in terms of performance and ease-of-use (particularly for non-scientific users).

The Geonamica software environment attempts to find a middle ground between these extremes by supporting (and even promoting) a highly modular implementation of models and allowing the incorporated model components and the links between them to be fully specified in run-time (i.e. without software development). Where extra flexibility is needed some components provide the possibility to script essential parts of a model (e.g. the formula used to calculate a particular variable can be entered in the user interface). The macro-economic model component in Metronamica even allows the entire model to be specified through the user interface. However, black box models are still supported by wrapping them as a single model component. The fact that we can thus integrate black box models with declarative models in one system allows us to make ad-hoc trade-offs between flexibility, performance and ease-of-use.

\subsection{Models}

Reusing models or model components is efficient and generally also improves the quality of models as they are tested various times in different contexts. Reusing models has proven most efficient when models are developed in small but meaningful model blocks. This allows reusing some blocks and adapting those for which the context is different in a different application. We found that properly documenting models, providing training on them and offering maintenance and support also has enhanced their reusability and uptake in new contexts. When incorporated in a generic ISDSS it is important that this system offers users sufficient flexibility through the GUI to tune the models to the specifics of their region of interest..

\subsection{User Interface}

The development of a user-friendly system is a formidable task, particularly when requirements are unclear. While reuse of user interface components saves time and can improve the quality of an ISDSS, it can only get us so far in terms of user-friendliness. It is much more beneficial to have a reusable structure for the user interface that directs towards an easy-to-use system, because this can take much longer to design than it takes to implement. The key to finding such a structure lies in a proper identification of the system's users, or rather the types of use of the system. As explained in section 2.2, Metronamica and tailor-made systems based on it support two types of users: modellers and policy analysts. Both are supported with a different structure that has shown to work well for the specific requirements that stem from either type of use.

\section{DISCUSSION AND CONCLUSIONS}

Based on the development and use of the generic Metronamica ISDSS and a number of tailor-made systems we have learned the following lessons:

\section{ISDSS development is a joint effort of users, scientist and IT-specialists}

The ISDSS design and development process benefits from a close interaction between the different parties involved: users, scientists, and IT-specialists. Understanding the needs of the various parties is a crucial task in developing a useful and usable ISDSS and often requires a number of iterations. These iterations can be included within projects and/or during follow-up projects with new or existing user groups. Iterations have played a crucial role in the development of the user interface, models and underlying software environment of Metronamica. Sometimes these developments have been demand-driven and other times supply-driven.

\section{In ISDSS development, software development is facilitating, not leading}

The main aim of ISDSS is to provide support to a decision process. Starting from this aim, the scientific and software requirements are derived. As many ISDSS are developed as part of research and development projects in which novel software solutions are sought, there is a risk to make this a leading force and the way forward to solve all problems. We caution therefore that good software solutions do not solve all conceptual challenges regarding model integration. Also issues related to usefulness and usability cannot be solved by technical solutions alone.

\section{The need for software development in various phases of the development and use of an ISDSS can and should be minimized}

We have shown that by developing a generic system that includes a range of configurable components, it is possible to set up applications to new regions without further software development. After applications have been set up, users still have a wide range of options to update data, set parameters and create indicators relevant for the study of interest. This approach greatly enhances the potential user group of these systems as 
programming skills and access to source code are no longer required in their application and use. Furthermore substantial costs are saved as no time needs to be spent on software development.

\section{Reusable concepts and components allow for efficient development of new ISDSS, although genericity remains a trade-off}

Reusability can be found in various forms: through the design process, the software environment, the models and the user interface design. The development of tailor-made systems can be made more efficient and effective by following a repeatable methodology that includes knowledge gained from previous projects and hence provides guidance and check lists during the entire process.

Reusing and further developing the same software environment for all ISDSS applications has been very efficient compared to starting from scratch for each new application. An important aspect of this is the fact that the software environment is not just a library of reusable components, but is a working application that can be customised with specific models and user interface elements. This allows the entire application logic to be reused, tested and enhanced in the development of each application. Developing a complimentary model library provides additional flexibility and scope over time as the model library expands with new components. Reusing models has proven most efficient when models are developed in small but meaningful model blocks. This allows reusing some blocks and adapting those for which the context is different in a different application.

Nonetheless we express some caution in developing fully generic components during the initial development stages of ISDSS as there is a trade-off between the level of detail and the genericity of a component. Moreover, in our experience it often only becomes clear after a first specific implementation what elements could and should be made generic to obtain a wider applicability. When the aim for reusability is not well balanced with a pragmatic attitude and real-world use, there is a risk that we merely achieve a theoretical possibility of reuse, because practical issues are not addressed.

\section{Funding mechanisms should be accounted for in planning long-term ISDSS development and implementation trajectories}

The funding for the Metronamica ISDSS comes from a combination of research projects, client-driven tailormade products and license fees, with desired enhancements carefully planned as part of these funding sources. Development of new generic components through a series of tailor-made ISDSS has proven to be a feasible development trajectory. Although we have often sought for opportunities in which various users could collaboratively finance developments that would be desirable for all, we have only sporadically been able to set up such projects. Difficulties in the co-development of components by various groups are mostly due to the sources of funding and the disabilities of users to maintain a budget for development. For this reason most of the co-development takes place during iterations in a series of projects financed by different funders. This has the advantage that (initial) components are available before improvements take place, but also the disadvantage that it is not possible to understand the user requirements of the wider user group during the first version of the respective component.

In our experience funding for software development is easier to obtain than funding for calibration and implementation of systems in user organisations. However, it is often a rather large step from a first prototype tailor-made ISDSS to a fully calibrated model that can be used for policy support, and without its actual use these systems will not be maintained. We therefore advice a development that puts sufficient attention to the usefulness, usability and model behaviour of the system and are careful to incorporate additional model components and stimulate theoretical and innovative software solutions without first testing these in practical applications.

\section{REFERENCES}

Barredo, J., Lavalle, C, Demicheli, L., Kasanko M., and McCormick, N. (2003). Sustainable urban and regional planning: The MOLAND activities on urban scenario modelling and forecast. Office for Official Publications of the European Communities, Luxembourg.

De Kok, J.L., and Wind, H.G. (2002). Designing rapid assessment models of water systems based on internal consistency. Journal of Water Resources Planning and Management, 128, 240-247.

Engelen, G., Uljee, I., and Van de Ven, K. (2003). WadBOS: integrating knowledge to support policymaking in the Dutch Wadden Sea. In: Geertman, S., and Stillwell, J. (Eds) Planning Support Systems in Practice, Springer-Verlag, Berlin, 513-537.

Fotheringham, A.S., Champion, T., Wymer, C., and Coombes, M. (2000). Measuring destination attractivity: a migration example. International Journal of Population Geography, 6, 391-421. 
Van Delden and Hurkens, A generic ISDSS for urban and regional planning

Fotheringham, A.S., and O'Kelly, M.E. (1989). Spatial Interaction Models. Kluwer, Dordrecht, the Netherlands.

Huser, B., Rutledge, D.T., Van Delden, H., Wedderburn, M.E., Cameron, M., Elliott, S., Fenton, T., Hurkens, J., McBride, G., McDonald, G., O’Connor, M., Phyn, D., Poot, J., Price, R., Small, B., Tait, A., Vanhout, R., and Woods, R.A. (2009). Creating futures: towards an integrated spatial decision support system for local government in New Zealand. In: Anderssen, R.S., Braddock, R.D., and Newham, L.T.H. (Eds), 18th IMACS World Congress - MODSIM09 International Congress on Modelling and Simulation. Cairns, Australia. ISBN: 978-0-9758400-7-8.

Hurkens, J., Vanhout, R., and Van Delden, H. Geonamica: software environment for model integration and DSS development, submitted to Environmental Modelling and Software.

Lavalle, C., McCormick, N., Kasanko, M., Demicheli, L., and Barredo, J.I. (2003). Monitoring and forecasting the dynamics of European urban areas - the territorial approach as key for urban development strategies. Urbanistica, 121, 105-111.

Muetzelfeldt, R. and Massheder, J. (2003). The Simile visual modelling environment. European Journal of Agronomy, 18, 345-358.

Muetzelfeldt, R. (2004). Declarative modelling in ecological and environmental research, Report EUR 20918, Luxembourg: Office for Official Publications of the European Communities, 85 pp, ISBN 92-8945212-9. http://www.simulistics.com/files/documents/dmeer.pdf

Ortuzar, J., and Willumsen, L.G. (1994). Modelling Transport, 2nd edition. John Wiley and Sons, Chichester, UK.

Oxley, T., McIntosh, B., Winder, N., Mulligan, M., and Engelen, G. (2004). Integrated modelling and decision-support tools: a Mediterranean example. Environmental Modelling and Software, 19, 999-1010.

RIKS, (2011). Metronamica - model descriptions. RIKS, Maastricht, The Netherlands: www.metronamica.nl

Rutledge, D.T., Cameron, M., Elliott, S., Fenton, T., Huser, B., McBride, G., McDonald, G., O’Connor, M., Phyn, S., Poot, J., Price, R., Scrimgeour, F., Small, B., Tait, A., Van Delden, H., Wedderburn, M.E., and Woods, R., (2008). Choosing regional futures: challenges and choices in building integrated models to support long-term regional planning in New Zealand. Regional Science Policy \& Practice, 1, 85-108.

Sieber, S., Zander, P., Verburg, P.H., and Van Ittersum, M. (2010). Model-based systems to support impact assessment - methods, tools and applications. Ecological Modelling, 221, 2133-2135.

Van Delden, H., Luja, P., and Engelen, G. (2007). Integration of multi-scale dynamic spatial models of socioeconomic and physical processes for river basin management. Environmental Modelling and Software, 22, 223-238.

Van Delden, H., Vanhout, R., Te Brömmelstroet, M., and White, R. (2009a). Design and development of Integrated Spatial Decision Support Systems: applying lessons learnt to support new town planning. In: Stolk, E., and Te Brömmelstroet, M. (Eds), Model Town: Using Urban Simulation in New Town Planning. SUN, Amsterdam, the Netherlands.

Van Delden, H., Kirkby, M.J., and Hahn, B.M. (2009b). Towards a modelling framework for integrated assessment in arid and semi-arid regions. In: Anderssen, R.S., Braddock, R.D., and Newham, L.T.H. (Eds), Proceedings of the 18th World IMACS Congress and MODSIM09 International Congress on Modelling and Simulation. Modelling and Simulation Society of Australia and New Zealand and International Association for Mathematics and Computers in Simulation, Cairns, Australia, ISBN: 978-09758400-7-8.

Van Delden, H., Van Vliet, J., Navarro, C., and Gutiérrez, E. (2010a). The Xplorah SDSS - supporting integrated planning on the island of Puerto Rico. 10th International Conference on Design \& Decision Support Systems in Architecture and Urban Planning, Eindhoven, the Netherlands.

Van Delden, H., Stuczynski,T., Ciaian, P., Paracchini, M.L., Hurkens, J., Lopatka, A., Shi,Y., Gomez Prieto, O., Calvo, S., Van Vliet, J., and Vanhout, R. (2010b). Integrated assessment of agricultural policies with dynamic land use change modelling. Ecological Modelling, 221, 2153-2166.

Van Delden, H., McDonald, G., Shi, Y. Hurkens, J., Van Vliet. J., and Van den Belt, M. (2011a). Integrating socio-economic and land-use models to support urban and regional planning. Proceedings of the 14th AGILE conference, April 18-21, Utrecht, the Netherlands.

Van Delden, H., Seppelt, R., White, R., and Jakeman, A.J. (2011b). A methodology for the design and development of integrated models for policy support. Environmental Modelling and Software, 26, 266279.

Van Ittersum, M.K., Ewert, F., Heckelei, T., Wery, J., Alkan Olsson, J., Andersen, E., Bezlepkina, I., Brouwer, F., Donatelli, M., Flichman, G., Olsson, L., Rizzoli, A., Van der Wal, T., Wien, J.E., and Wolf, J. (2008). Integrated assessment of agricultural systems - a component-based framework for the European Union (SEAMLESS). Agricultural Systems, 96, 150-165. 
Van Vliet, J., Navarro, C., Gutiérrez, E.R., Hurkens, J., and Van Delden, H. (2009). Xplorah Municipio, a multi resolution spatial decision support system. In: Haunert, J.-H., Kieler, B., and Milde, J. (Eds), Proceedings of the 12th AGILE International Conference on Geographic Information Science. IKG, Leibnitz Universität, Hannover, Germany, ISBN 2073-8013.

Veldkamp, A., and Lambin (2001). Predicting land-use change. Agriculture, Ecosystems \& Environment, 85, $1-6$.

Verburg, P.H., Schot, P.P., Dijst, M.J., and Veldkamp, A. (2004). Land-use change modeling: current practice and research priorities. GeoJournal, 61, 309-324.

White, R., and Engelen, G. (1993). Cellular automata and fractal urban form: a cellular modelling approach to the evolution of urban land use patterns. Environment and Planning A, 25, 1175-1199.

Zeigler, B., Praehofer, H., and Kim, T-G. (2000). Theory of Modeling and Simulation. Academic Press, New York. 